\title{
The Modeling Frame and Generation Flow of Combat Course of Action
}

\author{
Wan Lujun ${ }^{1, a}$, Li Wen ${ }^{2, b}$ and Huang Aqian ${ }^{3, c}$ \\ ${ }^{1}$ No.1\# Chang-le East Road Xi'an City, Shanxi Province, China \\ ${ }^{2}$ No.1\# Chang-le East Road Xi'an City, Shanxi Province, China \\ ${ }^{3}$ No.1\# Chang-le East Road Xi'an City, Shanxi Province, China \\ apndawlj@126.com, b1590306922@163.com, 472611184@qq.com
}

\begin{abstract}
Keywords: Joint Air Operations, Course of Action, Probabilistic Graphical Efficiency Validation, Graph Theory, Cascading Failure
\end{abstract}

\begin{abstract}
The course of action (COA) generation is the course of designing the task planning flow, which is one of the key supporting techniques while the military organization executing aerial operation. The high dynamic air battle field required the fast generation and regular updating with the change of the situation. Firstly, the concept and research process of COA is introduced, combining with the basic planning process of joint air operations, the joint air operations COA generation flow is provided with four steps. Due to the core problem of the process of action is to choose the best combination of actions based on alternative action plans, the COA modeling solution framework is established, one module is the establishment of the mobile network model, another module is to choose the optimal plan among all the feasible combinations described in module one, and the third module is the test of the action process.
\end{abstract}

\section{Concept and Research Progress}

The Course of Action (COA) is a type of overall orderly behavior that the joint commanding organization of arms of the services conducts rational planning of the combat troops' action, allocation of the task sequence, scheduling of military resources, and thus to achieve its operational objectives according to the analysis results of the battlefield-related information; it is a crucial component in the joint operational plan[1]. The generation of COA is a stochastic programming problem and a constant "optimization- feedback- optimization" process that requires modeling and optimization of changing battlefields and discrete random events. Usually the "effect-based" approach is adopted to solve complex problems in the battlefield, which often involves the problems of two aspects[2]: first, it is the program of action, which studies COA generation and optimization issues; second, it is combat planning, which satisfies the optimal allocation of resources of the implementation plan of the action program.

Because the action plan is essentially an optimal process for COA to achieve the overall mission, it can be seen as a directed evolution process with the goal of combat mission, meaning that according to the combat process, select a series of COA so that the strength of the organization or cluster can achieve the desired evolutionary results. Probabilistic network can represent the interrelationships between variables in a simple and effective way, and is becoming a powerful tool with broad application prospects in the uncertainty reasoning system[3]. In the application of COA generation, the probability network has prominent advantages in describing the association relations of COA elements, so it has gradually become the main tool for COA modeling and production in recent years. Probably the most representative of the probabilistic network is Bayesian Networks (BN) and Influence Networks (IN). BN, as a kind of directed probability network, is a probabilistic method which can be used to express uncertainty in the decision field. Later, through the simplification of knowledge representation, reasoning and learning, its computational complexity has been greatly reduced; usability and practicability have been greatly enhanced[4]. In the actual practice of COA problem modeling, BN has two limitations; one is probabilistic reasoning difficulties and the other one is the need to specify a large number of conditional probabilities. The former limitation leads to 
low time efficiency of variable probability calculation and the latter limitation leads to the complex modeling under a small amount of information[5]. IN simplifies the process of enlightening knowledge, and is ideal for conditional probability modeling between node variables that require subjective estimation. Standard IN can only describe the static causal relationship between random variables, but fail to give a valid description of the condition that the impact of variables has certain certain time-dependent relationship. Therefore, many scholars have improved the standard IN and influenced intensity parameter by introducing the specific time delay parameter, memory ring and non-stationary impact intensity parameter; they have proposed Timed Influence Nets[6], Dynamic Influence Nets[7], Activation Timed Influence Nets[8], thus further improving the description of action sequence on event impact in COA modeling.

Through the above analysis, the application of the probability graph model is more and more effective, in which the use of BN and IN is the most efficient. However, the former needs to solve the knowledge acquisition portfolio explosion problem; comparatively, IN has more advantages in terms of modeling method and knowledge representation. It not improves the time efficiency of calculating the variable probability, but also reduces the complexity of modeling,. It is one of the key ways that can be focused on in the future.

\section{Generation Process of COA}

Combining with the basic planning process of joint air operations, this paper will divide the generation of joint air operations COA into the following four steps: to analyze and clarify the key objectives of the enemy operations, to develop key action nodes and associated paths, to conceive multiple action plans and to optimize the best action process and test action process.

Analyze the Key Objectives of the Enemy Combat. The mission of joint air operations in the combat level is to combat the enemy's key objectives through carefully planned combat operations and to ultimately achieve the purpose of the war. In order to carry out a strong blow to the enemy's key objectives, it is necessary to analyze related data based on the joint battlefield intelligence analysis and task analysis, prove and confirm the correctness of the judgment of the enemy's key objectives. Generally, simply using air power to directly combat enemy targets is not the most effective method of warfare. In order to effectively combat the enemy's key objectives, it is necessary to conceive effective methods of warfare so as to influence the enemy's key objectives to the maximum. As the focus of the target is often an important goal that the enemy protects, so in the development of operational plans, the choice of goals is not necessarily directed at the key objectives, but to choose the associated factors or goals that combat the function exertion of key objectives. This requires to conduct the step-by-step analysis of the key objectives after selecting the key objectives in order to refine key vulnerabilities that affect key objectives and achieve the desired results by combating these critical vulnerabilities.

Under the premise of considering possible enemy action programs, major operational objectives and operational attempts, a direct or indirect approach may be used to carry out a rough operational conceptual design. Rough combat ideas usually include the commander's focus on the operational objectives, the completion time of the tasks, as well as the bottom line of the task risks that can be born. Rough operational concept is the basis for the subsequent formation of detailed action programs. Multiple feasible action programs can be formed for use according to the time requirements and energy of the staff.

Develop Key Action Nodes and Associated Paths. In the process of generating the COA, the decision maker should consider when, where, and how to use the combat power to combat the enemy's key objectives through key action nodes. The development of key action nodes is a true reflection of the staff's intellectual thinking ability in the decision-making process of combat operations and has a significant impact on the enemy, his own side or the battlefield environment. It is particularly important that each key action node contains a number of combat missions, actions and events that need to be clearly defined for its purpose, action and effectiveness, and quantified from the time domain, airspace and frequency domain. The purpose of the key action node must be clear, 
and can be measured by a certain assessment of the indicators. In the joint operations, the key action node will be basically the same as prewar expectations, which requires interrelated key action between the nodes to do the corresponding bedding. The key action nodes use the serial number, basic description, influencing factors, tasks and the formal requirements of the necessary resources and potential tasks to ensure that all operational goals and tasks and influencing factors can be associated with the action node.

Associated path of combat action node refers to actions that are connected and acted on different operational nodes to accomplish combat missions and achieve operational goals. Through the interaction of different action nodes, the associated path ensures that the combat tasks are completed in line with a certain logical order. In short, the associated path is that the combat forces use various action nodes in time and space. Each action node has multiple permutations, and some action nodes must be completed for multiple times in the associated path. Based on a number of key action nodes, different associated paths can be formed, but these different key paths are designed around the key objectives of combating the enemy. The difference is: combat direction, maneuvering speed, cost ratio and acceptable risk etc.

In the process of developing the associated path and conceptual action plan, it is necessary to face and analyze the choice of various accidental conditions, which can be expressed by branching and intermittent. On the associated path, the choice of branch or intermittent starting point is the commander's determination. Based on these determination points, the commander decide whether the combat process stops, continues or turns to the new process. A branch is a key action node that moves to an expected action node on an associated path, providing a more flexible initiative for the commander. The branch is only temporarily offset on an associated path and then back to the original associated path. Intermittent means that combat operations are clearly diverted to different affiliated paths and lead to new action programs. A determination point is a decision at a time or space on the associated path that the commander has an impact on a particular operational area. A determination point represents the action measures that the commander wishes to take, or the new action measures that the operational conditions decide to take.

Think of Multiple Action Plans and Optimize Them. Once the sorting of the key action nodes is completed, the action plan concept is about to begin. Whether the key action node is set up through the direct or indirect method, the enemy's possible action plans, operational targets and operational attempts must be attempted. When the combat action is divided into multiple stages, the staff member transforms each mission-critical node into a combat mission and translates the associated path into an action process. The operational tasks are mainly determined by space (geography, depth, front and rear regions) and time (combat phase), which should be spatially distinguished and distributed and arranged according to time.

The main contents of the relatively complete action plan include: operation objective and target; compilation, deployment of operation strength and weapon distribution; tasks of the troops, time and space(area, direction) of the main operational action; key objectives of the operation in the stages, the main operation method (including the combat style, the maneuvering style and the means of warfare), the probability of damage that may be achieved against the target, and so on. For air operations, it may also be divided into the strength of the airport configuration, dispatch time, formation methods, routes and a high degree of choice, weapons mounted, assault or interception tactics.

Verify the COA Process. Since COA is done with the commander's instructions and even direct involvement, test of COA is to see if it meets the commander's operational intent and meets the operational mission requirements. The unselected action plan should not be easily neglected, which can be used as the branch or intermittent of a planned scheme or applied as the fraud and wrestling scheme. The test procedure for COA process is as follows:

First of all, applicability. Does the action process meet the superiors' intentions? Has it finished all the tasks? What is the oddity of success? Does the action follow the superior operational instructions and the relevant operational principles? Does the COA meet the the needs of the task by the subjective assessment and can it complete the special, potential and important combat missions? 
Second, feasibility. Is there enough time and means to implement the program? The feasibility of the program can be analyzed from the following questions: whether there is sufficient time to complete the operational scenario, whether there is sufficient capacity in the space to carry out operations, whether there is sufficient force to carry out combat operations, and finally whether the action process takes into account all the constraints.

Third, acceptability. Evaluate the acceptability of COA by comparing risks and possible operational results. The risks include: the risk of COA, the extent to which the opponent can predict the program, and the extent to which the probability of success will be reduced.

Fourth, maintainability. The maintainability of the operational process can be evaluated in depth, front and rear regions at each stage of the war. Does the staff officer provide sufficient time for the troops to prepare and deploy? Is there enough time for the troops to reorganize to carry out the next mission? Is logistics support and operational costs reasonable?

Fifth, distinguishability. There must be a significant difference between the two action programs, each of which can be chosen by the commander and has a certain comparative advantage. For example, does each action plan differ only in branch and intermittent? Are there any other options for completing the task?

\section{Generation Modeling Framework of COA}

The whole process of action can be divided into multiple stages of action; the various stages of action are trying to control and change the combat environment state, and then transfer to the desired target state. In the process of changing the operational environment, the process of enemy action is also trying to influence the state of the environment through a series of actions. The presence of enemy action makes the degree of final effect different for different action processes facing the same operational objective. It can be seen that the core problem of the process of action is to choose the best combination of actions based on alternative action plans, such as the greatest probability of bringing the battle to the desired operational goal. The action process is focused on whether the operational objectives can achieve the desired effect by performing sequence actions and meet a set of constraints. In this way, the process of generating the process is divided into three modules, one module is the establishment of the mobile network model, that is, the battlefield environment between the qualitative and quantitative relationship between the elements; another module is to choose the optimal plan among all the feasible combinations described in Module One; the third module is the test of the action process, and its sensitivity analysis. In the first module, the probability of the enemy action is simulated by Monte Carlo randomly, and the probability of reaching the desired operational target is calculated by using the probability graph model based on the influence intensity. In the second module, according to the joint probability value, the intelligent optimization algorithm is adopted to optimize the combination of the action process. In the third module, the optimal action process is evaluated based on the performance test method, and the performance process is analyzed. The model's overall generation and solution framework is shown in Fig 1.

\section{Establishment of Action Network Model}

(1) Make Clear the Combat Objectives, Determine the Evaluation Index

The aim of making clear the combat goal is to set the desired effect of the mission task in advance, including the analysis of the effect and cost of earnings, comparison and sorting; on this basis, establish the evaluation index function of COA and conduct fitness assessment accordingly to this.

(2) Select the Optimization Algorithm and Parameters, Conduct Initialized Generation of Solution Space

According to the characteristics of the model and the comparative advantages of the algorithm, an algorithm with high computational efficiency and fast convergence is selected and the initial parameters of the algorithm are set. Under the conditions of time limit and resource constraints, according to the coding characteristics of the concrete algorithm, conduct initialized generation of the feasible solution space of COA, as a subset of solution space. T initial solution space should have as diverse characteristics as possible. 


\section{Optimization of Feasible Action Combinations Based on Maximum Joint Probability}

(1) Optimize Iterative Calculations

The iterative calculation process and the termination cycle of different algorithms may not be the same, but the goal is based on the deep search of the feasible solution space. If the feasible solution of the final finding is not in the initial solution space, the optimization process is the transfer process of the solution space, meaning that the feasible solution space transfers to the optimal solution orientation.

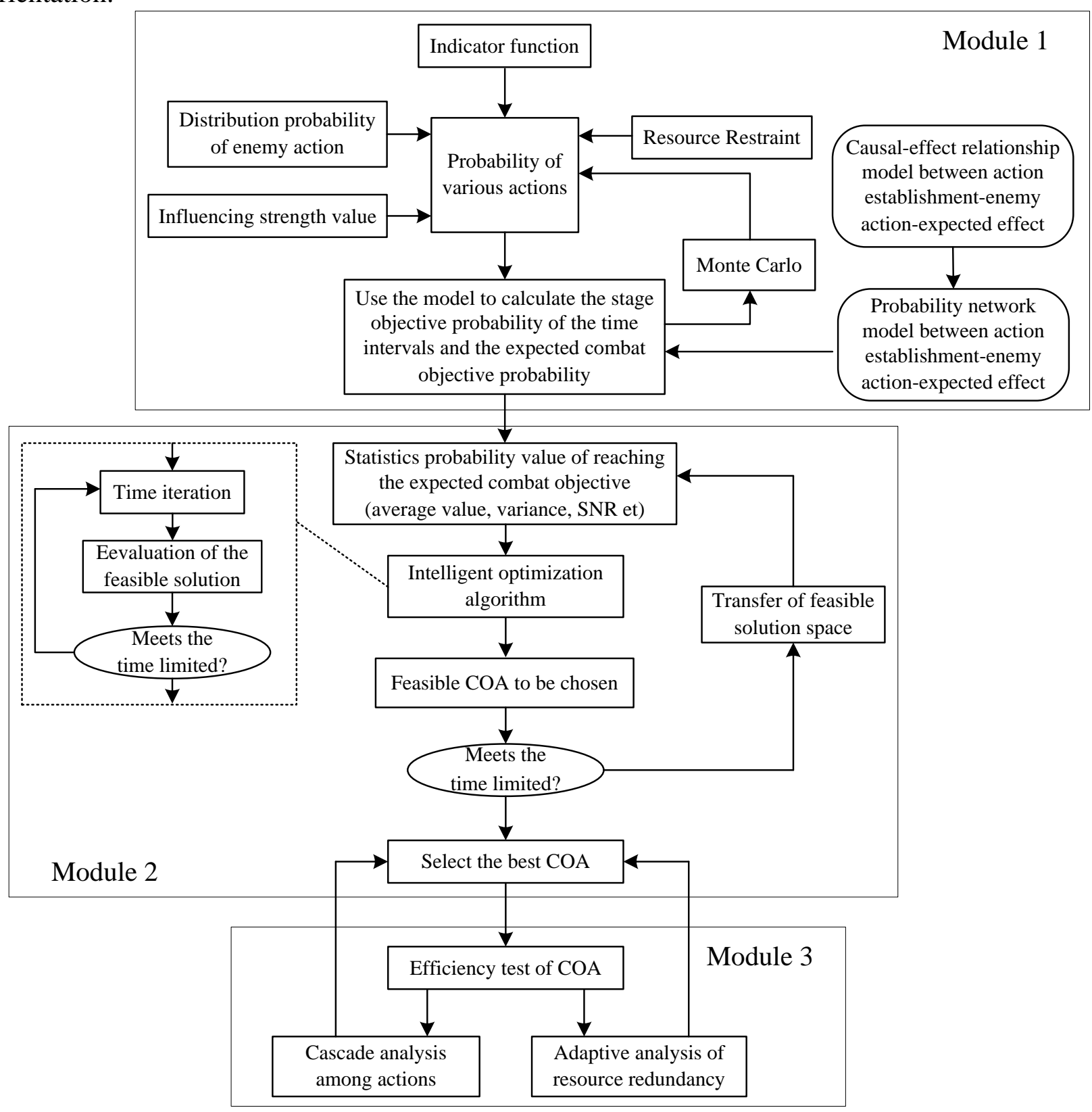

Fig 1 Modeling Solution Framework of COA

(2) Select the Solution to Be Evaluated

The convergence of the algorithm is directly determined by the choice of the solution to be evaluated. The algorithm with good performance can reduce the number of evaluations to a certain extent. In this way, the feasible solution quality of the solution space should be ensured in the process of calculation and synchronized with the iterative calculation of the algorithm.

(3) Evaluating Feasible Solutions and Sorting Merits

The evaluation index is used to evaluate the fitness function. According to the previously set indicators, the parameters are sorted by the parameters, and a feasible solution is obtained. The optimal solution is finally preserved. In many cases, random test method should be used and a number of iterative calculations should be conducted so as to select the optimal solution of the average sense. 


\section{COA Inspection}

(1) COA Performance Test

From the point of view of satisfying the basic requirements of combat effectiveness, the process of the optimal action is tested. This process needs to meet the logic expansion requirement of probabilistic graph reasoning.

(2) Cascade Failure Analysis

By analyzing the cascade failure of COA, it is possible to analyze the fragile sources of some or whole action sequence diagrams and the cascade failure paths that may be caused by fragile existence.

(3) Adaptive Analysis of Resource Redundancy

The appropriate redundancy protection of resources can improve the environmental adaptability of the generated COA. It is necessary to combine the certain theory to determine the range of resource redundancy, and realize the optimal balance of redundancy and adaptability.

\section{Summary}

As a systematic project, the formation of joint air operations COA should focus on the improvement of operational effectiveness and operational objectives. Based on the requirements of the integration of air operations in the future, this paper focuses on the basic theory and planned research generated by COA, centers on the characteristics of joint air operations in the context of information warfare; based on deep analysis of the COA concept and research of the progress situation, it sums up and abstracts the COA generation process "to analyze and clarify the key objectives of combat, to conceive key action nodes and associated paths, to conceive multiple action plans and to optimize the course of action and to test the action process." Aiming at the characteristics of high-speed dynamic and battlefield environment uncertainty, this paper constructs the key action path of generating causal relationship between causative relationship by using probability graph model, and then uses the optimization algorithm to get the basic framework for the best course of action.

\section{Acknowledgements}

This work was financially supported by the National Natural Science Fund (61472443, 61573017), Program of ShanXi Natural Science Foundation (2016JQ6062).

\section{References}

[1] Wu Yunpeng. Research of robust military action process program generation and optimization method[D]. Changsha: National University of Defense Technology, 2012.

[2] Boukhtouts A, Berger J, Guitouni A, et al. Description and analysis of military planning systems[R]. USA DRDC, 2005.

[3] Liu Jianwei, Li Haien, Luo Xionglin. Study on Probabilistic Graphic Learning Technology [J] .Journal of Software, 2014, 40 (6): 1025-1044.

[4] Guo Zhigao, Gao Xiaoguang, Di Ruohai. Self-adaptive parameter learning method of bayes network under small data collection conditions[J] .Control Theory Applications, 2016, 33 (7): 945-955.

[5] Lei T, Zhu C, Zhang W M. Dynamic bayes network based target-attacking course of action generation [C]. Proceedings of International Conference on Intelligence and Security Informatics, Changsha, 2013: 1-5.

[6] Haider S. From dynamic influence nets to dynamic Bayesian networks: a transformation algorithm[J]. International Journal of Intelligent Systems, 2010, 24(8):919-933. 
[7] Haider S, Levis A H. Modeling time-varying uncertain situations using dynamic influence nets[J]. International Journal of Approximate Reasoning, 2009, 49(2): 488-502.

[8] Zaidi A K, Papantoni K P. Theory of influence networks[J]. Journal of Intelligent \& Robotic Systems, 2010,60(3-4): 457-491. 